\title{
Short range correlations and wave function factorization in light and finite nuclei
}

\author{
C. Ciofi degli Atti, L.P. Kaptari, \\ H. Morita and S. Scopetta
}

Received: date / Accepted: date

\begin{abstract}
Recent BNL and Jlab data provided new evidence on two nucleon correlations $(2 \mathrm{NC})$ in nuclei. The data confirm the validity of the convolution model, describing the spectral function (SF) of a correlated pair moving in the mean field with high and low relative and center-of-mass $(\mathrm{cm})$ momenta, respectively. The model is built assuming that the wave function (WF) of a nucleus A, describing a configuration where the $\mathrm{cm}$ momentum of a correlated pair is low and its relative momentum is high, factorizes into the product of the two-body WF and that of the A-2 system. Such a factorization has been shown to occur in nuclear matter (NM). Here it is shown that few-body systems exhibit factorization, which seems to be therefore a general property, to be reproduced also in studies of the WF of finite nuclei.
\end{abstract}

Keywords Two nucleon correlations · Three- and four-body systems · Nuclear spectral function

A new generation of semi-inclusive and exclusive electron scattering experiments off nuclei is accounting for $2 \mathrm{NC}$ with unprecedented accuracy (see, e.g., Ref. [1]). This activity could have important implications for studies of cold dense nuclear matter. The nuclear SF is an important quantity for studies of $2 \mathrm{NC}$. For a nucleon with momentum $\mathbf{k}$ and removal energy $E$ in a nucleus $A$, the SF is defined as follows

$$
\begin{aligned}
P(k, E) & \equiv \frac{1}{2 J_{0}+1} \sum_{M_{0} \sigma}\left\langle\Psi_{A}^{0}\left|a_{\mathbf{k}, \sigma}^{\dagger} \delta\left[E-\left(H-E_{A}\right)\right] a_{\mathbf{k}, \sigma}\right| \Psi_{A}^{0}\right\rangle \\
& \equiv \frac{1}{2 J_{0}+1} \sum_{M_{0} \sigma} \sum_{f}\left|\left\langle\Psi_{A-1}^{f}\left|a_{\mathbf{k}, \sigma}\right| \Psi_{A}^{0}\right\rangle\right|^{2} \delta\left[E-\left(E_{A-1}^{f}-E_{A}\right)\right]
\end{aligned}
$$

Presented by S. Scopetta at the 21st European Conference on Few-Body Problems in Physics, Salamanca, Spain, 30 August - 3 September 2010

C. Ciofi degli Atti, L. P. Kaptari, S. Scopetta

Dipartimento di Fisica, Università degli Studi di Perugia and INFN, Sezione di Perugia, Italy

E-mail: ciofi@pg.infn.it; kaptari@pg.infn.it; scopetta@pg.infn.it

H. Morita

Sapporo Gakuin University, Japan E-mail: hiko@earth.sgu.ac.jp 

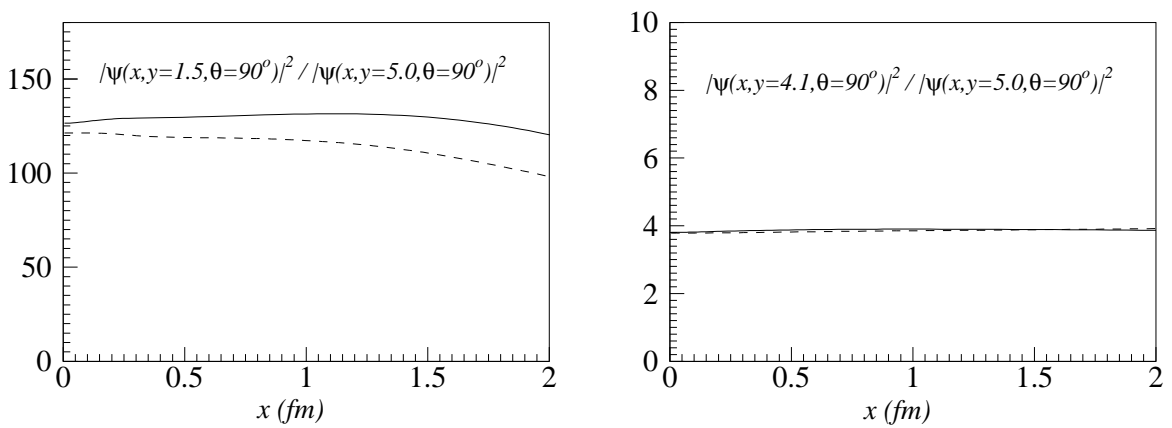

Fig. 1 The ratio Eq. (5) is shown for small values of $x$, for $\theta_{\widehat{\mathbf{x y}}}=90^{\circ}$ and for $y^{\prime}=5 \mathrm{fm}$. Left panel: $y=1.5 \mathrm{fm}$ (far from the $2 \mathrm{NC}$ region); right panel: $y=4 \mathrm{fm}$ (in the $2 \mathrm{NC}$ region) dashed line: the same ratio evaluated assuming that the $\mathrm{cm}$ motion is in $S$ wave.

It is clear from this definition that both the exact wave function (WF) of the ground state and the continuum WF of the $A-1$ system are necessary to evaluate the SF. These WF are very difficult to calulate, and models of the SF can be very helpful. $2 \mathrm{NC}$ are 2-body properties, while $P(k, E)$ is a 1-body quantity. Anyway, in the so called $2 \mathrm{NC}$ model [2, it is argued that, at high values of $k$ and $E, P(k, E)$ is dominated by ground-state configurations where the high momentum of one nucleun, $\mathbf{k}_{1} \equiv \mathbf{k}$, is entirely balanced by that of another one, $\mathbf{k}_{2} \simeq-\mathbf{k}$, while the remaining $(A-2)$ system has a momentum $\mathbf{k}_{A-2} \simeq 0$ 2. This yields

$$
P(k, E) \simeq P_{2 N C}(k, E)=\frac{1}{4 \pi} n(k) \delta\left[E-E^{(2 N C)}(k)\right]
$$

where $n(k)$ is the nucleon momentum distribution and energy conservation, $E_{A-1}^{*}+$ $\frac{k^{2}}{2 M_{A-1}}=\frac{k^{2}}{2 M}$, fixes $E^{(2 N C)}(k)=\left|E_{A}\right|-\left|E_{A-2}\right|+\frac{A-2}{A-1} \frac{k^{2}}{2 M}$. For these values of $E$, at high values of $k$, the exact nuclear SF of NM and ${ }^{3} \mathrm{He}$ show indeed a maximum, supporting the idea of the realization of a $2 \mathrm{NC}$-dominated configuration. The convolution model of Ref. [3] is a refined version of the $2 \mathrm{NC}$ one. At high values of $k$ and $E$, the two correlated particles move in the mean field created by the slow $(A-2)$ system. To model the SF, approximations are needed for the WF of the ground state and that of the $(A-1)$ system. As for the ground state WF, taking the motion of the $\mathrm{cm}$ of the pair in $\mathrm{S}$ wave $\chi_{0}(\mathbf{y})$, and the $A-2$ system in a low excitation state, $\Psi_{A-2}^{\overline{0}}\left(\left\{\mathbf{r}_{i}\right\}_{A-2}\right)$, yields the factorized expression

$$
\Psi_{A}^{0}\left(\left\{\mathbf{r}_{i}\right\}_{A}\right) \simeq \hat{\mathcal{A}}\left\{\chi_{0}(\mathbf{y})\left[\Phi(\mathbf{x}) \otimes \Psi_{A-2}^{\overline{0}}\left(\left\{\mathbf{r}_{i}\right\}_{A-2}\right)\right]\right\}
$$

As for the $A-1$ states, the interaction between the $A-2$ system and the correlated particles is neglected. Using these arguments, the spectral function turns out to be given by a convolution

$$
\begin{aligned}
P_{F N C}(k, E) & =\int d \mathbf{k}_{c m} \delta\left[E-\left|E_{A}\right|+\left|E_{A-2}\right|-\frac{(A-2)}{2 M(A-1)}\left(\mathbf{k}+\frac{(A-1) \mathbf{K}_{c m}}{(A-2)}\right)^{2}\right] \\
& \cdot n_{r e l}\left(\left|\mathbf{k}+\frac{\mathbf{K}_{c m}}{2}\right|\right) n_{c m}\left(\left|\mathbf{K}_{c m}\right|\right),
\end{aligned}
$$



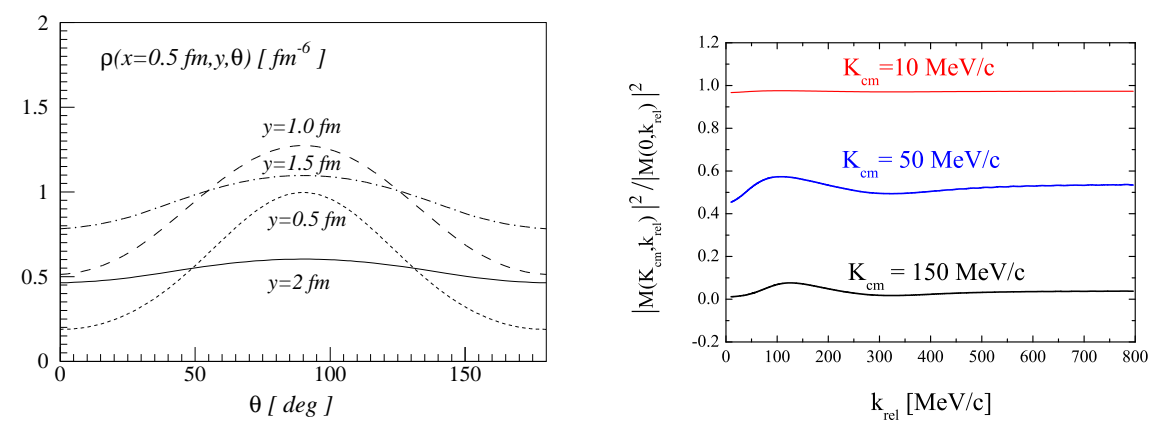

Fig. 2 Left panel: the quantity $\rho\left(x, y, \cos \theta_{\widehat{x y}}\right)$ (see text), for $x=0.5 \mathrm{fm}$, for four different values of $y$, as a function of $\theta_{\widehat{\mathbf{x y}}}$. Right panel: the ratio Eq. (9), as a function of $k_{r e l}$, for three different values of $\mathbf{K}_{c m}$.

where the argument of the $\delta$ function has been obtained from energy conservation, and $\mathbf{k}_{r e l}=\left(\mathbf{k}_{1}-\mathbf{k}_{2}\right) / 2, \mathbf{K}_{c m}=\mathbf{k}_{1}+\mathbf{k}_{2}=-\mathbf{k}_{3}$. One should notice that the simple $2 N C$ model is recovered placing $n_{c m}\left(\mathbf{k}_{c m}\right)=\delta\left(\mathbf{k}_{c m}\right)$, and that $P_{F N C}(k, E)$ is governed by the behaviour of the $\mathrm{cm}$ and relative momentum distributions, $n_{c m}$ and $n_{\text {rel }}$, respectively, whose calculation requires the knowledge of the ground-state wave function only. One should remember that, to evaluate the exact SF, the exact WF of the ground state, as well as the continuum WF of the $A-1$ system, are necessary. In the $2 \mathrm{NC}$ region, good agreement between $P_{F N C}(k, E)$ and the exact calculation for $\mathrm{NM}$ and ${ }^{3} \mathrm{He}$ is achieved 3]. Experimentally, a recent confirmation of the model has been obtained at BNL for ${ }^{12} C$ 4. Formally, the model has been justified for NM 5. As for Few Body Systems, the case of ${ }^{3} \mathrm{He}$ and ${ }^{4} \mathrm{He}$ is discussed here. More details will be shown in a forthcoming paper [6]. For the convolution model to hold, in the $2 \mathrm{NC}$ region, the WF has to factorize. For ${ }^{3} \mathrm{He}$, this means that, when $x=\left|\mathbf{r}_{1}-\mathbf{r}_{2}\right|<<y=\left|\mathbf{r}_{1}+\mathbf{r}_{2}\right| / 2, \Psi_{\frac{1}{2} \mathcal{M}}(\mathbf{x}, \mathbf{y}) \simeq \psi_{x}(\mathbf{x}) \cdot \psi_{y}(\mathbf{y})$ In the following, the occurrence of this property will be studied. Use will be made of the "Pisa" wave function [7, corresponding to the AV18 interaction [8]. If the $\mathrm{WF}$ factorizes in the $2 \mathrm{NC}$ region, also the quantity $\rho\left(x, y, \cos \theta_{\widehat{\mathbf{x y}}}\right)=\frac{1}{2} \sum_{\mathcal{M}}\left|\Psi_{\frac{1}{2} \mathcal{M}}(\mathbf{x}, \mathbf{y})\right|^{2}$ does. This means that this quantity has to depend weakly upon the angle $\theta_{\widehat{\mathbf{x y}}}$, and the dependence upon $|\mathbf{x}|$ and $|\mathbf{y}|$ can be separated. The formal expresion is cumbersom and will be shown elsewhere 6]. If the factorization holds in the $2 \mathrm{NC}$ region, one should have, for $x<<y, y^{\prime}$,

$$
R_{1}\left(x, y, y^{\prime}, \theta_{\widehat{\mathbf{x y}}}\right)=\frac{\rho\left(x, y, \cos \theta_{\widehat{\mathbf{x y}}}\right)}{\rho\left(x, y^{\prime}, \cos \theta_{\widehat{\mathbf{x y}}}\right)} \simeq \frac{f(y)}{f\left(y^{\prime}\right)}=\text { constant }
$$

This behavior, a clear signature of factorization, is found indeed around the $2 \mathrm{NC}$ region (see Fig. 1). It is also found that the $\mathrm{cm}$ motion is mainly in $\mathrm{S}$ wave (see Fig. 1) and that the relative motion is mainly deuteron-like [6]. Moreover, approaching the $2 \mathrm{NC}$ region, where $x<<y$, the dependence upon the angle $\theta_{\widehat{x y}}$ gets weaker and weaker, and the independence on the relative directions of $\mathbf{x}$ and $\mathbf{y}$ is obtained (see Fig.2, left 
panel). Let us discuss now the SF. In the convolution model, the SF for ${ }^{3} \mathrm{He}$ reads

$$
P_{F N C}\left(k_{1}, E\right)=\int d \mathbf{K}_{\mathbf{c m}} \delta\left[E-\mathcal{E}\left(\mathbf{k}_{\mathbf{1}}, \mathbf{K}_{\mathbf{c m}}\right)\right] n_{r e l}\left(\left|\mathbf{k}_{\mathbf{1}}+\frac{\mathbf{K}_{\mathbf{c m}}}{2}\right|\right) n_{c m}\left(\mathbf{K}_{c m}\right),(6)
$$

with $\mathcal{E}=\left|E_{3}\right|+\frac{1}{4 M}\left(\mathbf{k}_{\mathbf{1}}+2 \mathbf{K}_{\mathbf{c m}}\right)$, while the exact expression is found to be

$$
P\left(k_{1}, E\right)=\int d \mathbf{K}_{\mathbf{c m}} \delta\left[E-\mathcal{E}\left(\mathbf{k}_{\mathbf{1}}, \mathbf{K}_{\mathbf{c m}}\right)\right] \frac{1}{2} \sum_{\mathcal{S}=\mathcal{M}, S_{12}, \Sigma_{12}, \sigma_{3}}\left|\Phi_{\mathcal{S}}\left(\mathbf{K}_{c m}, \mathbf{k}_{r e l}\right)\right|^{2},
$$

where $\Phi_{\mathcal{S}}\left(\mathbf{K}_{c m}, \mathbf{k}_{r e l}\right)$ is the intrinsic ${ }^{3} \mathrm{He}$ wave function in momentum space. Comparing Eqs. (5) and (6) it is clear that, in the $2 \mathrm{NC}$ region, for the convolution model to hold, one has to find

$$
\frac{1}{2} \sum_{\mathcal{S}=\mathcal{M}, S_{12}, \Sigma_{12}, \sigma_{3}}\left|\Phi_{\mathcal{S}}\left(\mathbf{K}_{c m}, \mathbf{k}_{r e l}\right)\right|^{2} \simeq n_{c m}\left(\left|\mathbf{K}_{c m}\right|\right) n_{r e l}\left(\left|\mathbf{k}_{r e l}\right|\right) .
$$

The quantity $\left|\Phi_{\mathcal{S}}\left(\mathbf{K}_{c m}, \mathbf{k}_{r e l}\right)\right|^{2}$ has been studied. Approaching the $2 \mathrm{NC}$ region, where $\left|\mathbf{K}_{c m}\right|<<\left|\mathbf{k}_{r e l}\right|$, the dependence upon the angle $\theta_{\mathbf{K}_{\mathbf{c m}} \widehat{\mathbf{k}}_{r e l}}$ gets weaker and weaker. This behavior is the one to be studied in forth-coming experiments, measuring $n\left(\left|\mathbf{K}_{c m}\right|\right)$ and $n\left(\left|\mathbf{k}_{\text {rel }}\right|\right)$. Around the $2 \mathrm{NC}$ region, where $\left|\mathbf{K}_{c m}\right|<<\left|\mathbf{k}_{\text {rel }}\right|$ and the convolution model holds, one finds

$$
R=\frac{\left|\Phi\left(\mathbf{K}_{c m}, \mathbf{k}_{r e l}\right)\right|^{2}}{\left|\Phi\left(0, \mathbf{k}_{r e l}\right)\right|^{2}} \simeq \frac{n_{c m}\left(\left|\mathbf{K}_{c m}\right|\right)}{n_{c m}\left(\left|\mathbf{K}_{c m}\right|=0\right)}=\text { constant } \cdot n_{c m}\left(\left|\mathbf{K}_{c m}\right|\right)
$$

This allows one to see, for any $\left|\mathbf{K}_{c m}\right|$, at which value of $\left|\mathbf{k}_{r e l}\right|$ the convolution model starts to hold (see Fig. 2, right panel). Using this analysis, $n\left(\left|\mathbf{K}_{c m}\right|\right)$ and $n\left(\left|\mathbf{k}_{r e l}\right|\right)$ can be obtained in the $2 \mathrm{NC}$ region [6].

As for the factorization of the ${ }^{4} \mathrm{He} \mathrm{WF}$, encouraging preliminary results have been obtained using an ATMS wave function corresponding to the RSC interaction [9]. A result qualitatively similar to that of ${ }^{3} \mathrm{He}$ is found.

Summarizing, workable models of the SF, even if valid only in a peculiar region, can be very useful for phenomenological studies. The convolution model is a good approximation to the exact spectral function in the $2 \mathrm{NC}$ region. For ${ }^{3} \mathrm{He}$, it has been shown that, in the $2 \mathrm{NC}$ region, the exact wave function exhibits the factorization properties which justify the convolution model. A corresponding analysis is going on for ${ }^{4} \mathrm{He}$, and preliminary results are encouraging. These findings are useful also for finite nuclei: the factorization into $\mathrm{cm}$ and relative momentum distributions seems to be a general property in the $2 \mathrm{NC}$ region, and many-body calculations should reproduce it.

\section{References}

1. R. Subedi et al., Science 320, 1476 (2008).

2. L. Frankfurt and M. Strikman, Phys. Rep. 76, 215 (1981).

3. C. Ciofi degli Atti and S. Simula, Phys. Rev. C 53, 1689 (1996).

4. E. Piasetzky et al., Phys. Rev. Lett. 97, 162504 (2006).

5. M. Baldo et al., Nuc. Phys. A 604, 4298 (1996).

6. C. Ciofi degli Atti, L.P. Kaptari, H. Morita and S. Scopetta, in preparation.

7. A. Kievsky et al., Nuc. Phys. A 577, 511 (1994); Phys. Rev. Lett. 82, 3759 (1999).

8. R. Wiringa et al., Phys. Rev. C 51, 38 (1995).

9. H.Morita et al., Prog. Theor. Phys. 78 (1987), 1117. 